\title{
COVID19 Diagnosis using AutoML from 3D CT scans
}

This paper was downloaded from TechRxiv (https://www.techrxiv.org).

\section{LICENSE}

CC BY 4.0

SUBMISSION DATE / POSTED DATE

06-07-2021 / 10-07-2021

\section{CITATION}

Anwar, Talha (2021): COVID19 Diagnosis using AutoML from 3D CT scans. TechRxiv. Preprint. https://doi.org/10.36227/techrxiv.14914851.v1

$\mathrm{DOI}$ 


\title{
COVID19 Diagnosis using AutoML from 3D CT scans
}

\author{
Talha Anwar \\ Independent Researcher \\ chtal haanwaregmail.com
}

\begin{abstract}
Coronavirus is a pandemic that affects the respiratory system causing cough, shortness of breath, and death in severe cases. Polymerase chain reaction (PCR) tests are used to diagnose coronavirus. The false-negative rate of these tests is high, so there needs a supporting method for an accurate diagnosis. CT scan provides a detailed examination of the chest to diagnose COVID but a single CT scan comprises hundreds of slices. Expert and experienced radiologists and pulmonologists can diagnose COVID from these hundreds of slices, but this is very time-consuming. So an automatic artificial intelligence (AI) based method is required to diagnose coronavirus with high accuracy. Developing this AI-based technique requires a lot of resources and time, but once it is developed, it can significantly help the clinicians. This paper used an Automated machine learning (AutoML) technique that requires fewer resources (optimal architecture trials) and time to develop, resulting in the best diagnosis. The AutoML models are trained on $2 D$ slices instead of $3 D C T$ scans, and the predictions on unknown data (slices of CT scan) are aggregated to form a prediction of $3 D C T$ scan. The aggregation process picked the most occurred case, whether COVID or non-COVID from all CT scan slices and labeled the $3 D C T$ scan accordingly. Different thresholds are also used to label COVID or non-COVID $3 D$ CT scans from $2 D$ slices. The approach resulted in accuracy and F1-score of $89 \%$ and $88 \%$, respectively. Implementation is available at github.com/talhaanwarch/mia-covid19
\end{abstract}

\section{Introduction}

COVID-19 is a pandemic disease that mainly affects the respiratory system. The best way to prevent COVID is to isolate the infected peoples as it spread from patients to others very rapidly. This disease is usually diagnosed using reverse transcription-polymerase chain reaction (RT-PCR) test, but research showed that suspected patients should be examed with CT scans due to the high false-negative rate of PCR test. If a CT scan report is positive, no matter PCR is negative, the patient should not be removed from isolation [11]. Studies also showed that patients should not be diagnosed using CT scan only; instead, PCR test should also be performed to enhance the confidence level [11]. CT-scan can be used as a quick diagnostic method to categorize patients into "probably positive" and "probably negative" cohorts [2]. The bottom line is to use both PCR and CT-scan for the correct diagnosis of COVID patients.

Several studies have been performed to diagnose COVID from CT-scan images [1]. In this section, only studies performed on large public 3D CTscan datasets are discussed. Y. Qiblawey et al., trained segmentation models such as DenseNet 161 UNet and DenseNet201 FPN etc. on different datasets. Then these pre-trained models were used on Mosmeddata to get the lesions. Finally, the infected percentage is calculated from the lesions to diagnose COVID [12]. D. Kollias et al., introduced the MIA-COV19 (COV19-CT-DB) dataset and achieve a baseline macro F1score of $70 \%$ using 3D CNN-RNN network [10]. W.Tan and J.Liu used 3D CNN with BERT to extract features and MLP classifiers to classify the data. The authors used the COV19-CT-DB data and achieve an F1-score of $89 \%$ [16]. The current paper discussed the AtuoML approach to diagnose COVID from CT-scan images.

\section{Methodology}

\subsection{Dataset}

The COV19-CT-DB database is used in this study and is composed of three sets i.e. sets training, validation, and test data [10]. There are 1560, 374, and 3455 3D scans in training, validation, and test set, respectively. Each 3D scan ranges from 50 to $7002 \mathrm{D}$ slices. Training data has 690 COVID cases and 780 non-COVID cases. Similarly, validation data has $1653 \mathrm{D}$ CT scans of COVID patients and 209 CT scans of non-COVID subjects. The test set has 3455 3D CT scans with unknown labels that need to be predicted. The dataset is labeled by four specialists, two radiologists, and two pulmonologists instead of relying on PCR tests. 


\begin{tabular}{|c|l|l|l|}
\hline Data & & Train set & Validation set \\
\hline \multirow{2}{*}{ COVID } & 2D slice & 153681 & 35016 \\
\cline { 2 - 4 } & 3D image & 690 & 165 \\
\hline \multirow{2}{*}{ Non COVID } & 2D slice & 181991 & 40516 \\
\cline { 2 - 4 } & 3D image & 780 & 209 \\
\hline
\end{tabular}

Table 1. Number of 2D and 3D images in train and validation data

\subsection{Deep Learning}

Instead of using a 3D convolution neural network (CNN), 2D CNN is used to classify COVID vs. non-COVID cases. 2D CNNs are used because of limited GPU resources, as 3D CNNs require a high-end GPU system for training. Second, the slices in 3D images are not of the same length. It ranges from 50 to 700 slices in each 3D scan. So to use 3D CNNs, CT scans need to be truncated or padded. There are chances of noise addition while padding and information loss while truncating the data. So, 2D CNNs are trained on slice level instead of 3D volume level. Evaluation is made on slice level as well as the $3 \mathrm{D}$ volumetric level of validation data. For 3D level prediction, predictions are made on 2D slices, and then most occurred predictions are taken and assigned as 3D image labels. For example, if a CT-scan has 100 slices, 51 are predicted as COVID and 49 as non-COVID, the 3D image is labeled COVID. The threshold level technique is also applied. For example, if $1 \%$ of slices are predicted as COVID, the CT-scan is labeled as COVID. The threshold value of $1 \%, 5 \%, 10 \%$. 20\%, $30 \%, 40 \%$, and $50 \%$ are used to classify the validation data, and the threshold value that yielded the best results is used to produce test predictions.

\subsection{AutoML}

AutoML powered by AutoGluon is used to carry out the experiments for the classification of COVID vs. nonCOVID 3D ct-scans [3]. AutoML makes the classification pipeline automatic, avoiding the hustle of preprocessing and hyper-parameters tuning. For experimentation, Tesla P100 GPU is used. The batch size is set to 16 and the learning rate to 0.01 . The number of epochs is set to 15 , but if training time reached 4 hours, training is stopped. This helps to train more models in less time. The only preprocessing applied is to resize all images to $224 * 244$.

\subsection{Deep learning Architectures}

Different 2D CNN pre-trained models are used such as

\subsubsection{VGG}

VGG is one of oldest deep learning architecture proposed on 2014 and has a depth ranging from 16-19 [15]. VGG19_bn is 19th layers modified architecture with batch normalization layer added [14]. The concept of batch nor- malization is introduced in 2015, and almost all deep learning architectures after that used this layer [9]. Batch normalization layers reduce training time and overfitting issues of deep architectures.

\subsubsection{ResNet}

ResNet family of deep learning architectures came out in 2015 [4]. ResNet152 has dept up to 152 layers, but its complexity is quite less as compared to VGG models. ResNet introduced a concept called a shortcut connection that skips one or more layers and helps to avoid vanishing gradient problems caused by deeper length.

\subsubsection{DenseNet}

Almost after eight months of ResNet, DenseNet models were introduced [8]. DensetNet used a block called a Dense block in which a layer is connected to all subsequent layers in that block. DenseNet is deeper and has three times fewer parameters than ResNet.

\subsubsection{MobileNet}

MobileNet models were basically introduced for mobile devices with low computational powers in 2017 [6]. MobileNet is based on depth-wise separable convolution. It is a combination of depth-wise convolution and separable convolution. In this approach, $1 \times 1$ convolution is applied to all channels of input data. MobileNetV3 introduced in 2019 used AutoML to create best possible architecture [5]. It also used Squeeze and Excitation Networks (SENet) introduced in 2017 [7]. Squeeze is global average pooling. Excitation is two fully connected layers having RelU in between and Sigmoid at the end.

\subsubsection{Se_ResNext}

ResNext is similar to ResNet [17]. In ResNet, channels size is reduced by using $1 \mathrm{x} 1$ by convolution layer, for example, from 256 to 64 and then back to 256. In ResNext, channels size is reduced from 256 to 32 features maps, each having four channels and then aggregated back to 256. Applying convolution of 4 channels is much cheaper than on 64 channels. Se_ResNext is introducing SENet architecture in ResNext.

\subsubsection{ResNest}

ResNet was introduced in 2020 and it is a modularized architecture, which applies channel-wise attention on different feature map groups. [18]. 


\subsection{External data}

A portion of large external data [13] is also used to test the generalization of the models. The data portion comprised of 9776 non-COVID and 2282 COVID slices of different CT scans.

\section{Results}

All the experimentation discussed below is performed using NVIDIA TESLA P100 GPU. Fig 1 shows macro F1score on validation data using different threshold levels. $1 \%$ mean that if $1 \%$ COVID slices are present, then assign 3D CT-scan image as COVID case. Similarly, with other threshold levels but in the max threshold technique, if the maximum number of predicted slices labels belongs to COVID, then the 3D scan is assigned COVID else nonCOVID. The worst validation score is obtained when a CT scan is labeled as COVID based on $1 \%$ occurrence of coronavirus diagnosed slices. Results are also poor when CT scan is predicted corona positive because of 5\%, and $10 \%$ slices are predicted as positive. Best results are obtained when the $3 \mathrm{D}$ scan is labeled positive based on $30 \%$ or above occurrence of slices positive.

Table 2 shows the accuracy, precision, recall, and F1 score obtained on the validation set. The highest macro F1score and accuracy of $85 \%$ is achieved in ResNest14 architecture for 2D image data. On 3D CT-scan, ResNest14 lead to the highest accuracy and F1-score of $89 \%$ and $88 \%$, respectively. The lowest score is obtained using DenseNet201, where the macro F1-score on the 2D slice level and 3D scan is $81 \%$ and $84 \%$, respectively. The lowest precision is $81 \%$ when MobileNetv 3 model is evaluated on 2D slices and the lowest recall is $81 \%$ using DenseNet 201 . Table 3 shows the model size on disk and time for train-

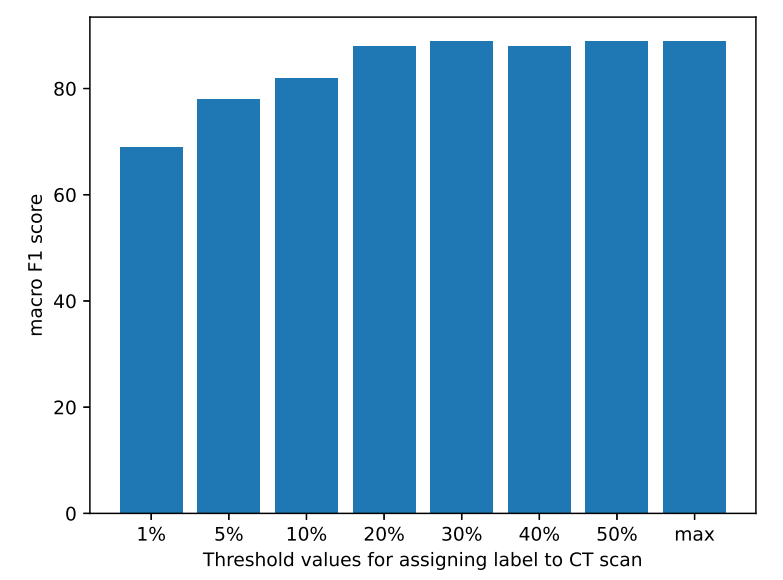

Figure 1. F1-score on validation data using different threshold values ing and inference on test set. Inference time is computed on test set having $3455 \mathrm{Ct}$-scans 3D images instead of validation set. MobileNetV3 has smallest models weights size and took around 6 hours 21 mutes for training and 2 hours 37 mutes for inference. VGG19 with batch normalization has the largest size of $1 \mathrm{~GB}$.

On external data macro F1 score of $76 \%$ and accuracy of $84 \%$ is achieved. Recall measures the model's ability to detect Positive samples and the model achieved a recall of $75 \%$

\section{Conclusion}

AutoML is the automatic way of doing any machine learning or deep learning task. We used the AutoGluon framework that leads to accurate diagnostic of COVID cased from 3D volumetric images. The advantage of $\mathrm{Au}-$ toGluon (AutoML) is that there is no need to worry about preprocessing, architecture implementation, and evaluation. It does all process internally and yield good results with hyper-parameters selected. Using ResNest14 architecture, accuracy and F1-score of $89 \%$ and $88 \%$ are obtained. Future work includes feeding input images of different sizes instead of fixed input of $224 * 224$. Fixing the input size for all models results in loss of information as different models learned differently on different input sizes.

\section{References}

[1] Talha Anwar and Seemab Zakir. Deep learning based diagnosis of covid-19 using chest ct-scan images. In 2020 IEEE 23rd International Multitopic Conference (INMIC), pages 1$5,2020$.

[2] Hester A Gietema, Noortje Zelis, J Martijn Nobel, Lars JG Lambriks, Lieke B Van Alphen, Astrid ML Oude Lashof, Joachim E Wildberger, Irene C Nelissen, and Patricia M Stassen. Ct in relation to rt-pcr in diagnosing covid-19 in the netherlands: a prospective study. PloS one, 15(7):e0235844, 2020.

[3] Jian Guo, He He, Tong He, Leonard Lausen, Mu Li, Haibin Lin, Xingjian Shi, Chenguang Wang, Junyuan Xie, Sheng Zha, et al. Gluoncv and gluonnlp: Deep learning in computer vision and natural language processing. J. Mach. Learn. Res., 21(23):1-7, 2020.

[4] Kaiming He, Xiangyu Zhang, Shaoqing Ren, and Jian Sun. Deep residual learning for image recognition. arxiv 2015. arXiv preprint arXiv:1512.03385, 2015.

[5] Andrew Howard, Mark Sandler, Grace Chu, Liang-Chieh Chen, Bo Chen, Mingxing Tan, Weijun Wang, Yukun Zhu, Ruoming Pang, Vijay Vasudevan, et al. Searching for mobilenetv3. In Proceedings of the IEEE/CVF International Conference on Computer Vision, pages 1314-1324, 2019.

[6] Andrew G Howard, Menglong Zhu, Bo Chen, Dmitry Kalenichenko, Weijun Wang, Tobias Weyand, Marco Andreetto, and Hartwig Adam. Mobilenets: Efficient convolutional neural networks for mobile vision applications. arXiv preprint arXiv:1704.04861, 2017. 


\begin{tabular}{|l|c|c|c|c|c|c|c|c|}
\hline & \multicolumn{4}{|c|}{ 2D CT-scan slice } & \multicolumn{3}{c|}{ 3D Volumetric Image } \\
\hline Model name & F1-score & Precision & Recall & Accuracy & F1-score & Precision & Recall & Accuracy \\
\hline ResNet152 & $80 \%$ & $82 \%$ & $80 \%$ & $81 \%$ & $85 \%$ & $89 \%$ & $85 \%$ & $86 \%$ \\
\hline DenseNet201 & $81 \%$ & $83 \%$ & $81 \%$ & $82 \%$ & $84 \%$ & $88 \%$ & $84 \%$ & $86 \%$ \\
\hline ResNest14 & $85 \%$ & $86 \%$ & $85 \%$ & $85 \%$ & $88 \%$ & $90 \%$ & $88 \%$ & $89 \%$ \\
\hline ResNext50 & $83 \%$ & $83 \%$ & $83 \%$ & $83 \%$ & $88 \%$ & $89 \%$ & $89 \%$ & $88 \%$ \\
\hline Se_ResNext50 & $84 \%$ & $84 \%$ & $83 \%$ & $84 \%$ & $87 \%$ & $89 \%$ & $87 \%$ & $88 \%$ \\
\hline MobileNetV3 & $80 \%$ & $81 \%$ & $88 \%$ & $81 \%$ & $86 \%$ & $88 \%$ & $86 \%$ & $87 \%$ \\
\hline VGG19_bn & $84 \%$ & $84 \%$ & $84 \%$ & $84 \%$ & $87 \%$ & $89 \%$ & $87 \%$ & $88 \%$ \\
\hline
\end{tabular}

\begin{tabular}{|l|l|l|l|}
\hline Model name & Model Size & Training Time & Inference Time \\
\hline ResNet152 & $472 \mathrm{MB}$ & 7 hours 41 minutes & 3 hours 15 minutes \\
\hline DenseNet201 & $166 \mathrm{MB}$ & 5 hours 21 minutes & 2 hours 48 minutes \\
\hline ResNest14 & $93 \mathrm{MB}$ & 7 hours 24 minutes & 2 hours 22 minutes \\
\hline ResNext50 & $203 \mathrm{MB}$ & 6 hours 31 minutes & 2 hours 7 minutes \\
\hline Se_ResNext50 & $222 \mathrm{MB}$ & 6 hours 10 minutes & 2 hours 49 minutes \\
\hline MobileNetV3 & $63 \mathrm{MB}$ & 6 hours 21 minutes & 2 hours 37 minutes \\
\hline VGG19_bn & $1 \mathrm{~GB}$ & 7 hours 56 minutes & 2 hours 42 minutes \\
\hline
\end{tabular}

Table 3. Model size and execution time. Infer time is on test set

[7] Jie Hu, Li Shen, and Gang Sun. Squeeze-and-excitation networks. In Proceedings of the IEEE conference on computer vision and pattern recognition, pages 7132-7141, 2018.

[8] Gao Huang, Zhuang Liu, Laurens Van Der Maaten, and Kilian Q Weinberger. Densely connected convolutional networks. In Proceedings of the IEEE conference on computer vision and pattern recognition, pages 4700-4708, 2017.

[9] Sergey Ioffe and Christian Szegedy. Batch normalization: Accelerating deep network training by reducing internal covariate shift. In International conference on machine learning, pages 448-456. PMLR, 2015.

[10] Dimitrios Kollias, Anastasios Arsenos, Levon Soukissian, and Stefanos Kollias. Mia-cov19d: Covid-19 detection through 3-d chest ct image analysis. arXiv preprint arXiv:2106.07524, 2021.

[11] Inan Korkmaz, Nursel Dikmen, Fatma Oztürk Keleş, and Tayibe Bal. Chest ct in covid-19 pneumonia: correlations of imaging findings in clinically suspected but repeatedly rt-pcr test-negative patients. Egyptian Journal of Radiology and Nuclear Medicine, 52(1):1-9, 2021.

[12] Yazan Qiblawey, Anas Tahir, Muhammad EH Chowdhury, Amith Khandakar, Serkan Kiranyaz, Tawsifur Rahman, Nabil Ibtehaz, Sakib Mahmud, Somaya Al Maadeed, Farayi Musharavati, et al. Detection and severity classification of covid-19 in ct images using deep learning. Diagnostics, 11(5):893, 2021.

[13] Mohammad Rahimzadeh, Abolfazl Attar, and Seyed Mohammad Sakhaei. A fully automated deep learning-based network for detecting covid-19 from a new and large lung ct scan dataset. Biomedical Signal Processing and Control, 68:102588, 2021.

[14] Marcel Simon, Erik Rodner, and Joachim Denzler. Imagenet pre-trained models with batch normalization. arXiv preprint arXiv:1612.01452, 2016.
[15] Karen Simonyan and Andrew Zisserman. Very deep convolutional networks for large-scale image recognition. arXiv preprint arXiv:1409.1556, 2014.

[16] Weijun Tan and Jingfeng Liu. A $3 \mathrm{~d}$ cnn network with bert for automatic covid-19 diagnosis from ct-scan images. arXiv preprint arXiv:2106.14403, 2021.

[17] Saining Xie, Ross B Girshick, Piotr Dollár, Zhuowen Tu, and Kaiming He. Aggregated residual transformations for deep neural networks. corr abs/1611.05431 (2016). arXiv preprint arXiv:1611.05431, 2016.

[18] Hang Zhang, Chongruo Wu, Zhongyue Zhang, Yi Zhu, Haibin Lin, Zhi Zhang, Yue Sun, Tong He, Jonas Mueller, R Manmatha, et al. Resnest: Split-attention networks. arXiv preprint arXiv:2004.08955, 2020. 